BNWL-363

\title{
PROMETHIUM ISOTOPIC POWER DATA SHEETS
}

RESEARCH

\section{and \\ DEVELOPMENT \\ REPORT}

\author{
H. T. FULLAM
}

H. H. VAN TUYL

FEBRUARY, 1967 


\section{LEGAL NOTICE}

This report was prepared as an account of Government sponsared work. Neither the United States, nor the Commission, nor any person acting on behalf of the Commission:

A. Makes any warranty or representation, expressed or implied, with respect to the accuracy, completeness, or usefuiness of the information contained in this report, or that the use of any information, apparatus, method, or process disclosed in this report may not infringe privately owned rights; or

B. Assumes any liabilities with respect to the use of, or for damages resulting from the use of any information, apparatus, method, or process disclosed in this report.

As used in the above, "person acting on behalf of the Commission" includes any employee or contractor of the Commission, or employee of such contractor, to the extent that such employee or contractor of the Commission, or employee of such contractor prepares, disseminates, or provides access to, any information pursuant to his employment or contract with the Commission, or his employment with such contractor.

\section{PACIFIC NORTHWEST LABORATORY}

RICHLAND, WASHINGTON

operated by

BATTELLE MEMORIAL INSTITUTE

for the

UNITED STATES ATOMIC ENERGY COMMISSION UNDER CONTRACT AT(45-1)-1830 
BNWL -363

UC-23, Isotopes

Industrial Technology

PROMETHIUM ISOTOPIC POWER

DATA SHEETS

By

H. T. Fullam

H. H. Van Tuy 1

Chemical Research Section

Chemistry Department

February 1967

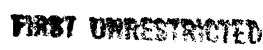

GITREBUTEN ?AADE

FEË24'67

PACIFIC NORTHWEST LABORATORY

RICHLAND, WASHINGTON 
Printed in the United States of America Available from

Clearinghouse for Federal Scientific and Technical Information National Bureau of Standards, U. S. Department of Commerce Springfield, Virginia 22151

Price: Printed Copy $\$ 3.00 ;$ Microfische $\$ 0.65$ 


\title{
PROMETHIUM ISOTOPIC POWER DATA SHEETS
}

by

\author{
H. T. Fullam and H. H. Van Tuyl
}

\section{INTRODUCTION}

The Division of Isotopes Development of the Atomic Energy Commission has assigned to Pacific Northwest Laboratory (Battelle-Northwest) the responsibility of developing technology for the preparation and use of promethium as a radioisotopic fuel in space, marine, and terrestrial power applications. Technology has been developed (and demonstrated on a several hundred gram scale) to permit large scale preparation of highly purified promethium. The half-life and specific heat generation rate were measured, and shielding requirements for promethium have been studied. The existence of ${ }^{146} \mathrm{Pm}$ in production and power fuels was discovered and its consequences investigated. Sources up to $60 \mathrm{~W}$ were prepared and are being investigated for long term stability. Several magacuries of promethium have been set aside from Purex process solutions for use in the development program.

Best estimates of the nuclear, chemical, and physical properties of promethium are summarized in the following pages. In many cases the chemical and physical properties have not yet been measured for promethium, but estimates have been made from measured values for neighboring rare earths. 


\section{DATA SHEET}

A. Fuel Form (as produced): Metal

1. Composition

Constituent
Promethium
Samarium
Neodymium
Yttrium
Lead
Aluminum

\section{Constituent}

$$
\begin{aligned}
& 146 \mathrm{Pm} \\
& 148 \mathrm{~m} \mathrm{Pm} \\
& { }^{154} \mathrm{Eu}
\end{aligned}
$$

$\frac{\text { Content, }}{\text { Minimum }} \underline{\text { Maximum }}$

Reference
99

$0.5 \quad 5$

0.52

02

0

$0 \quad 1$

Content, ppm by Activity

Minimum Maximum

$0.2 \quad 0.3$

$0 \quad 0.03$

$0.00001 \quad 0.01$

(Purity can be as high as $99.99 \%$ at time of separation, but ${ }^{147} \mathrm{Pm}$ decays to stable ${ }^{147} \mathrm{Sm}$ at the rate of $2.2 \% / \mathrm{mo}$.)

2. Specific Power (95 wt\% Pm)
a. $0.3164 \pm 0.0005 \mathrm{~W} / \mathrm{g}$
b. $882.4 \pm 1.7 \mathrm{Ci} / \mathrm{g}$

3. Radiation (Composition by Activity: $0.25 \mathrm{ppm}{ }^{146} \mathrm{Pm}, 0.025 \mathrm{ppm}{ }^{148} \mathrm{Pm}$, $0.001 \mathrm{ppm},{ }^{154} \mathrm{Eu}$ )

Energy,MeV Particles/W-sec $\quad \underline{\text { Source }} \quad \underline{\text { Reference }}$
a. Alpha
None
b. Beta
0.225
$1.03 \times 10^{14}$
$147 \mathrm{Pm}$
$3.1 \times 10^{9}$
${ }^{147} \mathrm{Pm}$
0.104
$9.0 \times 10^{6}$
$146 \mathrm{Pm}$
2.48
$1.0 \times 10^{5}$
${ }^{148} \mathrm{Pm}$
1.93
$2.6 \times 10^{4}$
${ }^{148} \mathrm{Pm}$ 


\section{Energy, MeV}

Beta

c. Gamma
1.02
0.71
0.52
0.42
1.855
0.87
0.58
0.26

\section{Energy, MeV}
0.121
0.75
0.45
1.465
1.015
0.916
0.914
0.727
0.630
0.611
0.601
0.551

0.502
0.433
0.414
$0.062-0.313$
1.61
1.276
1.006
0.997
0.874
0.758
0.724

Particles/W-sec

$7.7 \times 10^{4}$

$4.9 \times 10^{5}$

$6.2 \times 10^{5}$

$1.3 \times 10^{6}$

$1.0 \times 10^{4}$

$2.5 \times 10^{4}$

$3.9 \times 10^{4}$

$2.9 \times 10^{4}$

Photons/W-sec

$3.1 \times 10^{9}$

$1.7 \times 10^{7}$

$1.7 \times 10^{7}$

$5.2 \times 10^{4}$

$5.2 \times 10^{5}$

$4.6 \times 10^{5}$

$3.4 \times 10^{4}$

$8.5 \times 10^{5}$

$2.2 \times 10^{6}$

$1.5 \times 10^{5}$

$2.1 \times 10^{5}$

$2.4 \times 10^{6}$

$1.8 \times 10^{5}$

$1.5 \times 10^{5}$

$3.9 \times 10^{5}$

$7.2 \times 10^{5}$

$2.1 \times 10^{3}$

$3.9 \times 10^{4}$

$2.0 \times 10^{4}$

$1.1 \times 10^{4}$

$1.5 \times 10^{4}$

$2.1 \times 10^{3}$

$2.1 \times 10^{4}$
Source

$148 \mathrm{Pm}$

$148 \mathrm{~m} \mathrm{Pm}$

$148 \mathrm{~m} \mathrm{Pm}$

$148 \mathrm{~m}_{\mathrm{Pm}}$

$154 \mathrm{Eu}$

$154 \mathrm{Eu}$

${ }^{154} \mathrm{Eu}$

${ }^{154} \mathrm{Eu}$

Reference

Source Reference

${ }^{147} \mathrm{Pm}$

$146 \mathrm{Pm}$

$146 \mathrm{Pm}$

$148 \mathrm{Pm}$

$148 \mathrm{~m} \mathrm{Pm}$

$148 \mathrm{~m} \mathrm{Pm}$

$148 \mathrm{Pm}$

$148 \mathrm{~m} \mathrm{Pm}$

$148 \mathrm{~m}_{\mathrm{Pm}}$

$148 \mathrm{~m} \mathrm{Pm}$

$148 \mathrm{~m}_{\mathrm{Pm}}$

$148 \mathrm{~m} \mathrm{Pm}$,

$148 \mathrm{Pm}$

$148 \mathrm{~m} \mathrm{Pm}$

$148 \mathrm{~m} \mathrm{Pm}$

$148 \mathrm{~m} \mathrm{Pm}$

$148 \mathrm{~m} \mathrm{Pm}$

${ }^{154} \mathrm{Eu}$

${ }^{154} \mathrm{Eu}$

${ }^{154} \mathrm{Eu}$

${ }^{154} \mathrm{Eu}$

${ }^{154} \mathrm{Eu}$

$154 \mathrm{Eu}$

$154 \mathrm{Eu}$

(6) 


\begin{tabular}{|c|c|c|c|}
\hline \multirow{5}{*}{ Gamma } & Energy, Mev & Photons/W-sec & Source \\
\hline & 0.693 & $3.1 \times 10^{3}$ & \\
\hline & 0.593 & $6.2 \times 10^{3}$ & \\
\hline & 0.248 & $6.2 \times 10^{3}$ & \\
\hline & 0.123 & $3.3 \times 10^{4}$ & \\
\hline
\end{tabular}

$\underline{\text { Reference }}$

d. Brems-

$\begin{array}{cccc}\begin{array}{c}\text { Brems- } \\ \text { Strahiung }\end{array} & 0.22 & 2.6 \times 10^{4} & \begin{array}{l}147 \mathrm{Pm}, \\ 146 \mathrm{Pm}, \\ 148 \mathrm{Pm}\end{array} \\ 0.20 & 8.0 \times 10^{6} & \begin{array}{l}147 \mathrm{Pm} \\ 147 \mathrm{Pm}\end{array} \\ 0.18 & 9.4 \times 10^{7} & 147 \mathrm{Pm} \\ 0.16 & 4.8 \times 10^{8} & 147 \mathrm{Pm} \\ 0.14 & 1.7 \times 10^{9} & 147 \mathrm{Pm} \\ 0.12 & 4.8 \times 10^{9} & 147 \mathrm{Pm} \\ 0.10 & 1.2 \times 10^{10} & 147 \mathrm{Pm} \\ 0.08 & 3.0 \times 10^{10} & 147 \mathrm{Pm} \\ 0.06 & 7.4 \times 10^{10} & 147 \mathrm{Pm} \\ 0.04 & 2.0 \times 10^{11} & 147 \mathrm{Pm} \\ 0.02 & 7.2 \times 10^{11} & \end{array}$

e. Neutrons

None

\section{Half Lives}

$$
\begin{aligned}
& \frac{\text { Nuclide }}{147 \mathrm{Pm}} \\
& 146_{\mathrm{Pm}} \\
& 148 \mathrm{~m}_{\mathrm{Pm}} \\
& 154 \mathrm{Eu}
\end{aligned}
$$

Half Life

$$
\begin{aligned}
& 2.620+0.005 \mathrm{yr} \\
& 4.40 \pm 0.2 \mathrm{yr} \\
& 41 \mathrm{days} \\
& 16 \mathrm{yr}
\end{aligned}
$$

Since little information is available on promethium and its compounds, most of the data given in Sections 5, 6, 7 and 8 of the following tables were obtained by interpolation from existing data for neodymium, samarium and other rare earths. The data listed in brackets were obtained by interpolation.

5. Compatibility with Materials of Containment 
6. Physical Properties

$\underline{\text { Value }}$

$\underline{\text { Reference }}$

(Promethium meta1)

a. Density $\mathrm{g} / \mathrm{cm}^{3}$

7.3

b. Coefficient of linear

thermal expansion

$25^{\circ} \mathrm{C}$

$\left[6.7 \pm 0.4 \times 10^{-6}\right]$

Average -173 to $800{ }^{\circ} \mathrm{C}$

[8.6 $\times 10^{-6}$ ]

c. Heat capacity $\left(\mathrm{cal} / \mathrm{mole}^{\circ} \mathrm{C}\right) \quad 0-1035{ }^{\circ} \mathrm{C}$

$\left[C_{p}=6.5+0.0025 \mathrm{~T}\right]$

d. Melting Point $\left({ }^{\circ} \mathrm{C}\right)$

865

1080

e. Boiling Point $\left({ }^{\circ} \mathrm{C}\right)$

[3200]

f. Phase Transition Temperature $\left({ }^{\circ} \mathrm{C}\right)$

[890]

g. Heat of Phase Transition (kcal/mole)

[0.73]

$(10,11)$

h. Heat of Vaporization (kcal/mole)

[60]

i. Heat of Fusion (kcal/mole)

[1.88]

[1.95]

$(10,11,13)$

j. Vapor Pressure (mmHg)

$[\log \mathrm{P}=6.50-$

$\frac{13,000}{\mathrm{~T}}$ ]

k. Thermal Conductivity

$\left(\mathrm{cal} / \mathrm{sec}-\mathrm{cm}^{\circ} \mathrm{C}\right) 300^{\circ} \mathrm{K}$

[0.031]

$[0.179]$

1. Thermal Diffusivity

m. Viscosity

n. Surface Tension (dynes/cm) $2300{ }^{\circ} \mathrm{C}$

$[40-70]$

o. Total Hemispherical Emissivity

p. Spectral Emissivity

q. Crystallography BCC

$\mathrm{a}=7.60 \mathrm{~A}^{\circ}$

r. Solubilities

s. Diffusion Rates 
7. Mechanical Properties

(Promethium metal)

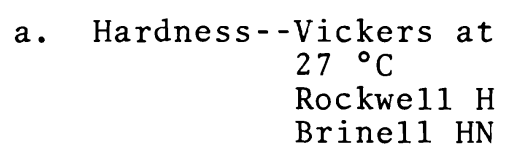

\begin{tabular}{lcl}
\multicolumn{2}{c}{ Value } & Reference \\
Cast & Cold Work & \\
{$[74]$} & {$[76]$} & $(19,20)$ \\
{$[56]$} & & $(13)$ \\
{$[45]$} & & $(11,13)$
\end{tabular}

b. Crush Strength

8. Chemical Properties

(Promethium metal)

a. Thermodynamic Functions
1. So (cal/mole-deg) $300^{\circ} \mathrm{K}$
[17.1]
2. $\frac{\mathrm{H}_{\mathrm{t}}^{\circ}-\mathrm{H}_{\mathrm{O}}^{\circ}}{\mathrm{F}_{\mathrm{t}}^{\circ}-\mathrm{H}^{\circ}}(\mathrm{cal} / \mathrm{mole}-\mathrm{deg}) 300{ }^{\circ} \mathrm{K}$
[6.07]
3. $\frac{\mathrm{F}_{\mathrm{t}}^{\circ}-\mathrm{H}_{\mathrm{O}}^{\circ}}{\mathrm{T}}(\mathrm{cal} / \mathrm{mole}-\mathrm{deg}) 300{ }^{\circ} \mathrm{K}$
[11.0]

(13)

b. Chemical Reactions

Promethium metal can be expected to react in the same general fashion as other rare earth metals. Typical reactions might include the following:

$$
\begin{array}{ll}
2 \mathrm{Pm}+3 / 2 \mathrm{O}_{2} & \stackrel{>25{ }^{\circ} \mathrm{C}}{\longrightarrow} \mathrm{Pm}_{2} \mathrm{O}_{3} \\
2 \mathrm{Pm}+3 \mathrm{~S} & \stackrel{500{ }^{\circ} \mathrm{C}}{\longrightarrow} \mathrm{Pm}_{2} \mathrm{~S}_{3} \\
\mathrm{Pm}+\mathrm{H}_{2} & \stackrel{>25{ }^{\circ} \mathrm{C}}{\longrightarrow} \mathrm{PmH}_{\mathrm{x}} \\
\mathrm{Pm}+1 / 2 \mathrm{~N}_{2} & \stackrel{900{ }^{\circ} \mathrm{C}}{\longrightarrow} \mathrm{PmN} \\
\mathrm{Pm}+3 \mathrm{H}_{2} \mathrm{O} & \longrightarrow \mathrm{Pm}(\mathrm{OH})_{3}+3 / 2 \mathrm{H}_{2}
\end{array}
$$

\begin{tabular}{|c|c|c|c|c|}
\hline & \multicolumn{2}{|c|}{$40 \mathrm{hr}$ Week } & \multicolumn{2}{|c|}{$168 \mathrm{hr}$ Week } \\
\hline & Air & Water & Air & Water \\
\hline Soluble & $6 \times 10^{-8}$ & $6 \times 10^{-3}$ & $2 \times 10^{-9}$ & $2 \times 10^{-4}$ \\
\hline Insoluble & $1 \times 10^{-7}$ & $6 \times 10^{-3}$ & $3 \times 10^{-9}$ & $2 \times 10^{-4}$ \\
\hline
\end{tabular}

9. Biological Tolerances

Maximum Permissible Concentration, $\mu \mathrm{Ci} / \mathrm{m} 1$ 
10. Shielding Data

Reference

Material

$\mathrm{U}$

$\mathrm{Pb}$

W

Mo

$\mathrm{Fe}$
Shielding for 100X Attenuation, $\mathrm{g} / \mathrm{cm}^{2}$

2.0 to 50

1.5 to 59

1.7 to 66

4.8 to 93

15 to 103

(Thickness varies with source size and shape, and amount of other external shielding)

Shield Thickness $\mathrm{cm}$ of Uranium
0.01
0.02
0.05
0.1
0.2
0.5
1
2

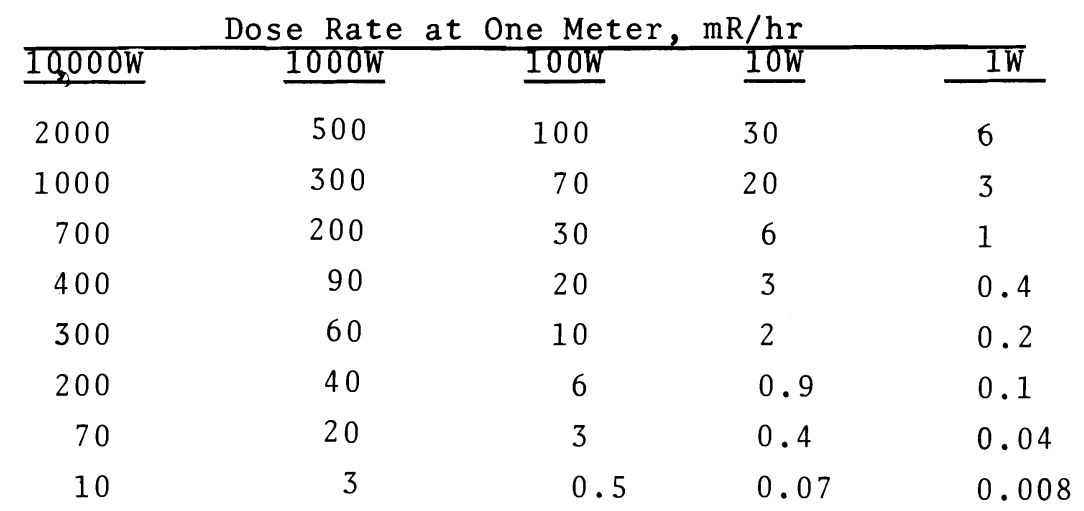

B. Fuel Form (as produced): Sesquioxide $\left(\mathrm{Pm}_{2} \mathrm{O}_{3}\right)$

1. Composition

Constituent
Promethium
Samarium
Neodymium
Yttrium
Lead
Aluminum
Oxygen

\section{Content, Wt:}

\begin{tabular}{cc}
\hline Minimum & Maximum \\
\cline { 2 - 2 } 81 & 85 \\
0.4 & 4 \\
0.4 & 1.7 \\
0 & 1.7 \\
0 & 1.7 \\
0 & 0.9 \\
14.0 & 14.7
\end{tabular}

$\underline{\text { Reference }}$ 
Content, ppm by Activity

Constituent $\quad$ Minimum

$\underline{\text { Reference }}$

(1)

2. Specific Power ( 82 wt: Pm)

a. $0.2731 \pm 0.0004 \mathrm{~W} / \mathrm{g}$

b. $761.6 \pm 1.4 \mathrm{Ci} / \mathrm{g}$

3. Radiation

Same as for ${ }^{147} \mathrm{Pm}$ metal.

4. Half Lives

Same as for ${ }^{147} \mathrm{Pm}$ metal.

Since little information is available on promethium and its compounds, most of the data given in Sections 5, 6, 7 and 8 of the following tables were obtained by interpolation from existing data for neodymium, samarium and other rare earths. The data listed in brackets were obtained by interpolation.

5. Compatibility with Materials of Containment $\left(\mathrm{Pm}_{2} \mathrm{O}_{3}\right)$

6. Physical Properties $\left(\mathrm{Pm}_{2} \mathrm{O}_{3}\right)$

$\underline{\text { Value }}$

$\underline{\text { Reference }}$
a. Density $\left(\mathrm{g} / \mathrm{cm}^{3}\right)$ A. $\mathrm{Pm}_{2} \mathrm{O}_{3}$
7.62
(Theoretical,
B. $\mathrm{Pm}_{2} \mathrm{O}_{3}$
7.43
X-ray data.)
C. $\mathrm{Pm}_{2} \mathrm{O}_{3}$
6.84

b. Coefficient of 1 inear

thermal expansion

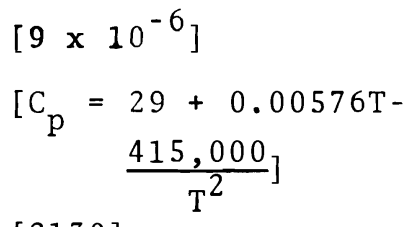

c. Heat Capacity $\left(\mathrm{ca} 1 / \mathrm{mole}-{ }^{\circ} \mathrm{C}\right)$

[2130]

d. Me1ting Point $\left({ }^{\circ} \mathrm{C}\right)$

[>3000]

e. Boiling Point $\left({ }^{\circ} \mathrm{C}\right)$ 
f. Phase Transition Temperature $\left({ }^{\circ} \mathrm{C}\right)$
C. $\mathrm{Pm}_{2} \mathrm{O}_{3} \rightarrow$ B. $\mathrm{Pm}_{2} \mathrm{O}_{3}$
$950{ }^{\circ} \mathrm{C}$
B. $\mathrm{Pm}_{2} \mathrm{O}_{3} \rightarrow$ A. $\quad \mathrm{Pm}_{2} \mathrm{O}_{3}$
$>1000^{\circ} \mathrm{C}$

g. Heat of Transition

h. Heat of Vaporization ( kcal/mole)

i. Heat of Fussion (kcal/mole)

j. Vapor Pressure $(\mathrm{mmHg})$ for Lanthanum $\left[\log \mathrm{P}=15.62-\frac{39,800}{\mathrm{~T}}\right]$

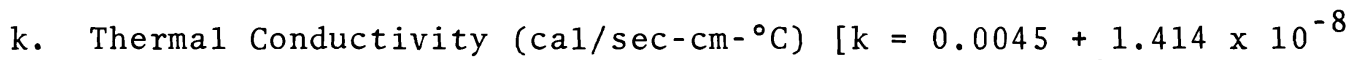
$\left.(1020-\mathrm{T})^{2}\right]$

1. Thermal Diffusivity

m. Viscosity

n. Surface Tension

o. Total Hemispherical Emmissivity

p. Spectral Emmisivity

q. Crystallography

A. $\mathrm{Pm}_{2} \mathrm{O}_{3}$ hcp. $\quad \mathrm{a}=3.806 ; \mathrm{c}=5.954$

B. $\mathrm{Pm}_{2} \mathrm{O}_{3}$ Monocl.

$\mathrm{a}=14.15 ; \mathrm{b}=3.69 ; \mathrm{c}=8.78$

C. $\mathrm{Pm}_{2} \mathrm{O}_{3}$ bcc

$a=10.99$

r. Solubilities in $\mathrm{H}_{2} \mathrm{O}$ Sol. Prod.

$1 \times 10^{-22}$ for $\mathrm{Pm}(\mathrm{OH})_{3}$

s. Diffusion Rates

7. Mechanical Properties $\left(\mathrm{Pm}_{2} \mathrm{O}_{3}\right)$
a. Hardness
b. Crush Strength 
8. Chemical Properties $\left(\mathrm{Pm}_{2} \mathrm{O}_{3}\right)$

a. Thermodynamic Properties

1. $\Delta \mathrm{H}_{\mathrm{f}}^{\circ} \mathrm{kcal} / \mathrm{mole}\left(298^{\circ} \mathrm{K}\right)$

2. $\mathrm{S}^{\circ} \mathrm{cal} / \mathrm{mole}-{ }^{\circ} \mathrm{C}\left(298^{\circ} \mathrm{K}\right)$

3. $\Delta \mathrm{F}_{\mathrm{f}}^{\circ} \mathrm{kcal} / \mathrm{mole}\left(298^{\circ} \mathrm{K}\right)$

b. Chemical Reactions

Promethium oxide can be expected to react in the same general fashion as the oxides of samarium and neodymium. Typical reactions might include:

$$
\begin{aligned}
& \mathrm{Pm}_{2} \mathrm{O}_{3}+6 \mathrm{HCl}_{(\mathrm{aq})}+2 \mathrm{PmCl}_{3} \cdot \mathrm{X} \mathrm{H}_{2} \mathrm{O} \\
& \mathrm{Pm}_{2} \mathrm{O}_{3}+6 \mathrm{HNO}_{3} \rightarrow 2{\mathrm{Pm}\left(\mathrm{NO}_{3}\right)_{3}} \\
& \mathrm{Pm}_{2} \mathrm{O}_{3}+\mathrm{HF} \stackrel{700{ }^{\circ} \mathrm{C}}{\longrightarrow} 2 \mathrm{PmF}_{3}+3 \mathrm{H}_{2} \mathrm{O} \\
& \mathrm{Pm}_{2} \mathrm{O}_{3}+7 \mathrm{C} \stackrel{>1000{ }^{\circ} \mathrm{C}}{\longrightarrow} 2 \mathrm{PmC}_{2}+3 \mathrm{CO}
\end{aligned}
$$

\section{Biological Tolerances}

Same as for ${ }^{147} \mathrm{Pm}$ metal.

10. Shielding Data

Within limits of reporting, the same as for ${ }^{147} \mathrm{Pm}$ metal. 


\section{REFERENCES}

1. F. P. Roberts and H. H. Van Tuyl. Promethium-146, Fission Product, and Transuranium Isotope Content of Power Reactor Fuels, BNWL-45. Pacific Northwest Laboratory, Richland, Washington. March 1965.

2. E. J. Wheelwright, D. M. Fleming, and F. P. Roberts. "Calorimetric Determination of the Mean $B$ Energy and Half Life of Promethium147", J. Phys. Chem., vol 69, p 1220. 1965 .

3. D. Stromminger, J. M. Hollander and G. T. Seaborg. "Table of Isotopes", Rev. Mod. Phys., vol 30, p 585. 1958.

4. E. G. Funk,Jr., J. W. Mihelich, and D. F. Schwerdtfeger. "Radioactive Decay of Pm-143, Pm-144 and Pm-146", Phys, Rev., vol 120, p 1781. 1960.

5. C. V. K. Baba, G. T. Ewan, and J. R. Svarez. "Level in Sm-148 Fed in the Beta Decay of Pm-148 and Pm-148m", Nucl. Phys., vol 43, p 264. 1963.

6. Nuclear Data Sheets, National Academy of Sciences, National Research Council, vol 5. April 1964.

7. H. H. Van Tuyl. BREMRAD - A Computer Code for External and Internal Bremsstrahlung Calculations, HW-83784, General Electric Company, Richland, Washington. Sept. 1964.

8. I. M. H. Pagden, R. Jakeways, and F. C. Flack. "The Decays of Pm-143, Pm-144 and Pm-146", Nucl. Phys., vol 48, p 555. 1963.

9. H. T. Fullam, L.J. Kirby, and R. T. AlZemann. Promethium Heat Source Compatibility Studies - Part I. Studies at $1100{ }^{\circ} \mathrm{C}, B N W L-327$, Pacific Northwest Laboratory, Richland, Washington. 1966. (Confidential)

10. F. H. Spedding and A. H. Daane. The Rare Earths, John Wiley \& Sons, New York. 1961.

11. E. M. Savitskii. Rare Earth Alzoys, AEC-Tr-6151, Washington, D. C., 1962 .

12. F. H. Spedding, J.J. Mckeown and A. H. Daane. "The High Temperature Thermodynamic Functions of Cerium, Neodymium and Samarium", J.Phys. Chem., vol 64, p 289. 1960. 
13. K. A. Gschneider, Jr. Rare Earth AlZoys, D. Van Nostrand Company, New York. $196 \overline{1 .}$

14. F. Weigel. Fifth Rare Earth Research Conference, Book 3 , Iowa State Univ. 1965.

15. K. A. Gschneider. "Physical Properties and Inter-relationships of Metallic and Semi-Metallic Elements", Solid state Physics, vol 16, p 275 (Ed. by F. Seitz and Turnbull) Academic Press, New York. 1964.

16. R. K. WiZZiams and D. L. McElroy. Estimated Thermal Conductivity Values for solid and Liquid Promethium, ORNL-TM-1424, Oak Ridge National Laboratory, Oak Ridge, Tenn. March 1966.

17. S. Legvold and F. H. Spedding. Quarterly Summary Research Report in Physics for Apriz, May and June, 1954, ISC-508, Ames, Iowa. August 1954.

18. H. H. Van Tuyl (Compizer). Promethium Isotopic Power Data Sheets, BNWC-45, Pacific Northwest Laboratory, Richland, Washington. March 1965.

19. A. C. Davis (Editor), Design Engineering, vol 60, p 163. october 1964.

20. E. V. Kleber and B. Love. The Technology of ScandiumYttrium and the Rare Earth Metals, MacMillan Company, New York, 1963 .

21. G. V. Samsonov. High Temperature Compounds of Rare Earth Metal with Nonmetals, Consultants Bureau, New York. 1965 .

22. Title 10, Code of Federal Regulations, Part 20, Appendix B. 1966 .

23. H. H. Van Tuyl, F. P. Roberts and E. J. Wheelwright. Shielding Requirements for Promethium Sources, HW-77375, General Electric Company, Richland, Washington. April 1963.

24. J. O. Blomeke and W. T. Ziegler. "The Heat Content, Specific Heat and Entropy of $\mathrm{La}_{2} \mathrm{O}_{3}, \mathrm{Pr}_{6} \mathrm{O}_{11}$ and $\mathrm{Nd}_{2} \mathrm{O}_{3}$

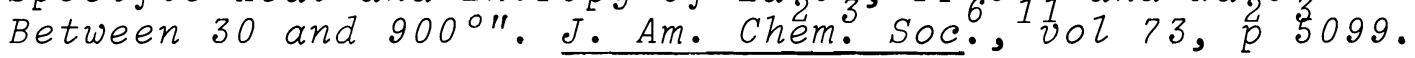
1951 .

25. S. J. Yosim and T. A. Milne. Basic Chemistry of High Temperature Inorganic Systems. Semiannual Progress Report, January-June, 1956, NAA-SR-1797, North American Aviation Inc., Downey, California. March 1957. 
26. W. E. Kingery and F. H. Norton. The Measurement of Thermal Conductivity of Refractory Materials, Quarterly Progress Report for the Period Ending July 1, 1955 , NYO

27. W. M. Latimer. Oxidation Potentials, Prentice Hall, Inc., Englewood, Cliffs, N.J. 1956.

28. J.A. Gibson, J. F. Mizzer, P. S. Kennedy, and G. W. P. Rengstorff. The Properties of the Rare Earth Metals and Compounds, Battelle Memorial Institute, Columbus, Ohio. 1959.

29. T. Chikalla. Unpublished Data, Battelle-Northwest, (Personal Communication) 1967. 


\section{$\underline{\text { DISTR I BUTION }}$}

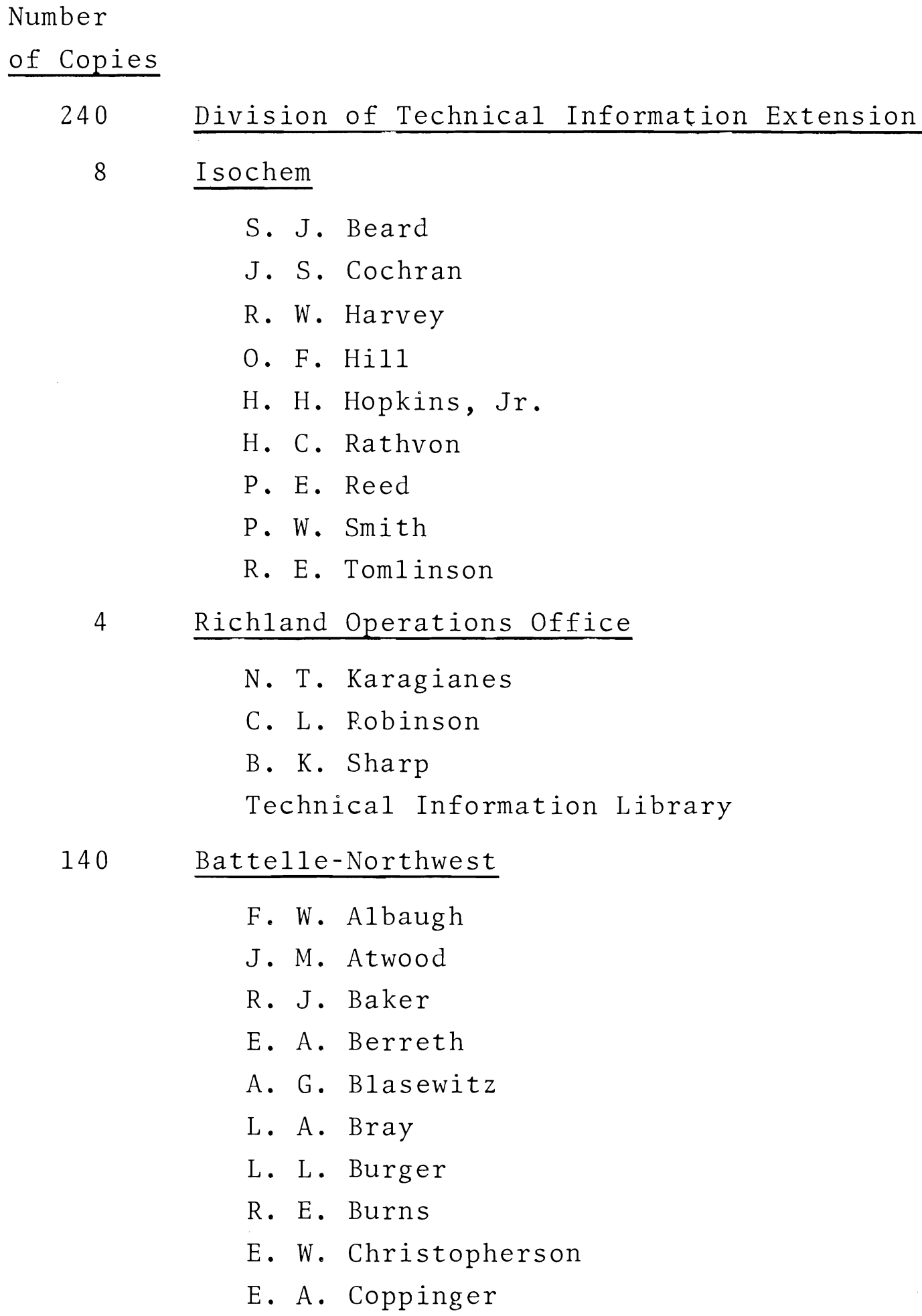


Number

of Copies

Battelle-Northwest (contd)

G. M. Dalen

D. R. de Halas

D. E. Deonigi

R. F. Dickerson

K. Drumheller

E. A. Eschbach

H. T. Fullam

K. M. Harmon

E. R. Irish

A. R. Keene

L. J. Kirby

R. W. McKee

R. L. Moore

J. M. Nielsen

H. M. Parker

R. S. Pau 1

A. M. Platt

F. P. Roberts

C. A. Rohrmann

R. C. Smith

U. L. Upson

H. H. Van Tuy 1

E. E. Voiland

M. T. Walling

E. J. Wheelwright

Technical Information Files

Technical Publications 


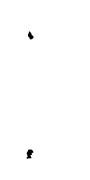

